Gut, 1982, 23, 630-632

Case report

\title{
Overwhelming pneumococcal septicaemia in a patient with ulcerative colitis and splenic atrophy
}

\author{
K J FOSTER, ${ }^{*} \mathrm{~N}$ DEVITT $\mathrm{P}$ J GALlagheR, and $\mathrm{R}$ M ABBOTT
}

From the Southampton University Hospitals, Southampton

SUMmaRY This report describes a young woman with ulcerative colitis who developed rapidly fatal pneumococcal septicaemia. Necropsy revealed splenic atrophy. This case supports the hypothesis that splenic atrophy might contribute to the morbidity of ulcerative colitis. The occurrence of splenic atrophy in ulcerative colitis is now well established. ${ }^{1}$ Splenic atrophy from other causes has been associated with severe bacterial infections, often pneumococcal. ${ }^{2}$ It has been suggested that splenic atrophy is most severe when ulcerative colitis is active and may contribute to postoperative morbidity. ${ }^{2}$ This case documents overwhelming septicaemia in a patient with splenic atrophy whose colitis was in remission.

\section{Case report}

A 22 year old English woman presented in 1977 with a three year history of intermittent bloody diarrhoea. Ulcerative colitis was diagnosed on the basis of typical clinical and sigmoidoscopic features, with histological evidence of severe non-specific colitis on rectal biopsy and total colitis on barium enema examination. She was initially treated with oral prednisolone and salazopyrine and, during exacerbations, with further short courses of steroids orally or by retention enema. At the time of her terminal illness her ulcerative colitis was symptomatically in remission. She had required treatment with prednisolone by enema and orally three months and seven months earlier respectively. Her maintenance therapy was salazopyrine $1 \mathrm{~g}$ twice daily, although she did not take this regulary.

In March 1981 the patient, her husband, and daughter developed mild diarrhoea, which they ascribed to 'food poisoning'. This caused little concern and husband and daughter rapidly recovered. The patient began to vomit and developed persistent headache. After 24 hours she suddenly collapsed. She was seen within half an hour by her general practitioner who noted that she was shocked and had a few purpuric spots. She was immediately transferred to hospital by which time

\footnotetext{
* Address for correspondence: Dr K J Foster, Royal United Hospital, Combe Park, Bath BA1 3NG.

Received for publication 20 November 1981
}

she was deeply shocked, cyanosed, and had a florid purpuric rash. Septicaemia was diagnosed but no source of infection was clinically evident. She was treated with intravenous fluids, hydrocortisone, penicillin, gentamycin, and metronidazole. Radiographs of the chest and abdomen were normal, sigmoidoscopy showed a slightly oedematous mucosa with multiple purpuric spots but the vascular pattern was preserved. Plasma urea, electrolytes, and amylase were normal except for a bicarbonate level of $13 \mathrm{mmol} / \mathrm{l}$. Her haemoglobin was $15 \mathrm{~g} / \mathrm{dl}$, white count $49 \times 10^{9} / 1$, and platelet count $55 \times 10^{9} / 1$. She died suddenly within three hours of her collapse at home after an apparently trivial gastrointestinal upset.

After her death blood cultures taken on admission produced a heavy growth of Streptococcus pneumoniae type 22. (Danish classification; typing was kindly performed by the Streptococcus Reference Unit, Central Public Health Laboratory, Colindale).

\section{NECROPSY REPORT}

At necropsy no primary focus of pneumococcal infection could be found. There was no mucosal ulceration of the small or large bowel, but there were numerous petechial haemorrhages on the mucosal surfaces. Both adrenal glands were diffusely haemorrhagic. The spleen was markedly atrophic and weighed $38 \mathrm{~g}$ (normal weight 150-200 g). 
HISTOLOGICAL APPEARANCES

Sections of colon and adrenals embedded in paraffin were treated with trypsin and stained by an unlabelled antibody peroxidase - antiperoxidase method using rabbit antihuman fibrin antibody. ${ }^{3}$ Sections were counterstained by haematoxylin or by Gram's method. Paraffin-embedded sections of spleen and lymph nodes were also examined after routine staining.

The colon and adrenal glands showed abundant fibrin deposition in many small capillaries, often in close association with large aggregates of Grampositive cocci (Fig. 1). There was extensive atrophy of both $\mathrm{T}$ and $\mathrm{B}$ cell areas of the spleen with preservation of the red pulp (Fig. 2). Histology of the lymph node was normal.

\section{DISCUSSION}

This patient's illness showed characteristic features ascribed to the syndrome of 'overwhelming postsplenectomy infection', including prodromal symptoms of malaise and vomiting, sudden collapse and shock, disseminated intravascular coagulation, and the Waterhouse-Friderichsen syndrome. Most often the infection is caused by Streptococcus pneumoniae, usually found in large numbers in the blood, and as many as $50 \%$ of patients die. ${ }^{2}$ In previous studies it has been suggested that splenic atrophy is related to the activity of the ulcerative colitis. ${ }^{1}$ Our patient was in remission from ulcerative colitis and there was no clinical or necropsy evidence of an alternative cause of splenic atrophy. Histological examination indicated that the atrophy affected $T$ and $B$ lymphocyte areas within the spleen

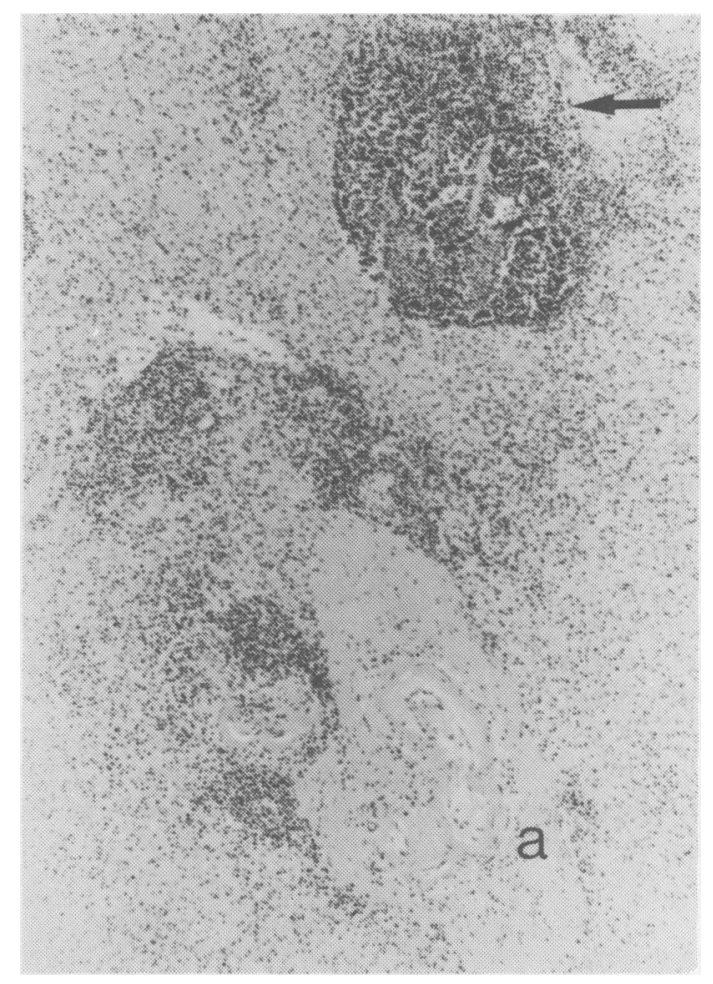

Fig. 2 Photomicrograph of spleen stained by haematoxylin and eosin $\times 110$. Although this is one of the most cellular areas there is marked lymphoid atrophy. Note absence of germinal centre formation in the B cell follicular area (arrow) and paucity of lymphoid tissue in $T$ cell periartereolar zone (a).

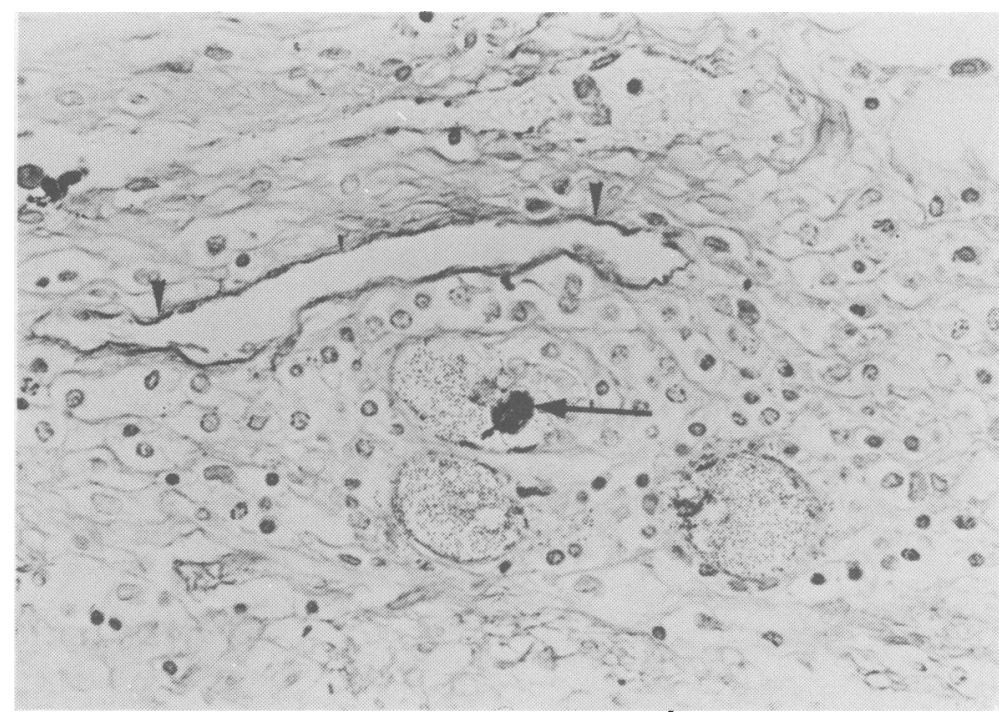

Fig. 1 Submucosa of caecum stained for fibrin (black) by immunoperoxidase technique. Gram counterstain $\times 420$. Note numerous cocci in small blood vessels. Fibrin is present among bacterial clumps (long arrow) and on the endothelial surface of a vein (arrow heads). 
but with sparing of the lymph nodes. It has recently been reported that splenic atrophy in patients with ulcerative colitis is not associated with generalised dysfunction of the reticuloendothelial system. ${ }^{4}$

One of the four hyposplenic patients developing septicaemia after colectomy described by Ryan et al also had pneumococcal infection. It has been suggested that polyvalent pneumococcal capsular protein vaccine is protective in children with splenic atrophy, at least in the short term. ${ }^{5}$ Others have suggested that the antibody response may be impaired in splenectomised patients, ${ }^{6}$ but one current recommendation ${ }^{7}$ would suggest that hyposplenism in patients with ulcerative colitis should be sought, possibly by isotope scanning, ${ }^{1}$ and immunisation offered. Although commercially available vaccine in the United Kingdom contains antigen from strains commonly causing septicaemia, it does not, however, contain the antigen type that killed our patient.

Dr J Bamforth kindly gave his permission for this case to be reported.

\section{References}

1 Ryan FP, Smart RC, Holdsworth CD, Preston FE. Hyposplenism in inflammatory bowel disease. Gut 1978; 19: $50-5$.

2 Krivit W. Overwhelming post splenectomy infection. American J Haematol 1977; 2: 193-201.

3 Mepham BL, Frater W, Mitchell BS. The use of proteolytic enzymes to improve immunoglobulin staining by the PAP technique. Histochem J 1979; 11: 345-57.

4 Palmer KR, Barber DC, Sherriff S, Holdsworth CD. Reticuloendothelial function in coeliac disease and ulcerative colitis. Gut 1981; 22: A419.

5 Ammann AJ, Addiego J, Wara DW, Lubin B, Smith WB, Mentzer WC. Polyvalent pneumococcal polysaccharide immunization of patients with sickle cell anaemia and patients with splenectomy. $N$ Engl $J$ Med 1977; 297: 897-900.

6 Hosea SW, Burch CG, Brown EJ, Berg RA, Frank MM. Impaired immune response of splenectomised patients to polyvalent pneumococcal vaccine. Lancet 1981; 1: 804-7.

7 Public Health Service Advisory Committee on Immunization Practices. Pneumococcal polysaccharide vaccine. Morbidity and Mortality Weekly Report 1978; 27: 25-31. 